\title{
Disk Clearing in the Young Binary AK Sco
}

\author{
Eric L. N. Jensen \\ Swarthmore College, Dept. of Physics \& Astronomy, \\ Swarthmore, PA 19081 USA
}

\begin{abstract}
The pre-main-sequence spectroscopic binary AK Sco is surrounded by a circumbinary disk. Yet while this disk must be somewhat disturbed by the binary at its center, there is no evidence from current data that it has a cleared central hole like those seen in some young binaries, nor that accretion from the outer disk has been cut off. Indeed, most of its spectral and photometric diagnostics are indistinguishable from those of young single stars. Why doesn't this system show any evidence of disturbance of its disk? It may be that the center of the disk is indeed largely cleared, but that a small amount of dust in this region supplies the observed near-infrared excess.
\end{abstract}

\section{Introduction}

It is now clear that most young, low-mass binary stars of all separations have associated disks, and that the disks can be circumstellar and/or circumbinary (see my other contribution to this volume). The simple, cartoon picture of the influence of a binary on its associated disks is that a gap (with zero surface density) is cleared in the region of the binary orbit, while the disks are relatively undisturbed both inside and outside the gap. The aim of this contribution is to provide evidence (albeit anecdotal) that the disk-binary interaction (and resultant disk geometry) is considerably more complicated than this simple picture would suggest.

AK Sco is a double-lined spectroscopic binary with a period of 13.6 days, F5 star components (masses of 1.05 and $1.07 M_{\odot} / \sin ^{3} i$ ), and a semi-major axis $(a \sin i$ ) of $0.14 \mathrm{AU}$ (Andersen et al. 1989). It lies in the Upper Sco subgroup of the Sco-Cen OB association at a distance of approximately 145 pc (Favata et al. 1998); its estimated age is $\sim 5 \times 10^{6}$ yr (Andersen et al. 1989).

\section{Optical Variability}

Like most T Tauri stars, AK Sco is variable (Figure 1). Andersen et al. (1989) pointed out that the system's optical colors tend to get redder as it gets fainter, and they suggested that this is consistent with changing obscuration of the system by clumps of circumstellar (or circumbinary) material. Detailed study of the system over many orbital periods has shown that the variability is aperiodic, and in particular that it is uncorrelated with the binary orbital period (Figure 1; Andersen et al. 1989; L. P. Vaz, personal communication). 




Figure 1. AK Sco is variable, but the variability is aperiodic and uncorrelated with the orbital phase, unlike the case of DQ Tau (Mathieu et al. 1997). Data courtesy of Luiz Paulo Vaz.

\section{Disk Properties}

AK Sco has a strong infrared excess, with IRAS and submillimeter fluxes suggesting a substantial disk, most of which must be circumbinary (Jensen \& Mathieu 1997, hereinafter JM97). However, unlike the young spectroscopic binaries V4046 Sgr and 162814-2427, AK Sco shows no lack of near-infrared excess indicative of a cleared hole in the center of the disk (JM97). Rather, it has a spectral energy distribution that is almost a power law in the infrared (Figure 2), consistent with a continuous disk.

The two stars pass within about eight stellar radii of each other at closest approach in their orbit (Andersen et al. 1989), leaving little room for circumstellar disks. Taking 0.2 times the binary separation as a typical circumstellar disk radius in an eccentric binary (Artymowicz \& Lubow 1994), these disks would only extend to about three stellar radii around each star. Such small disks might be expected to be rapidly depleted by accreting onto the stars, yet the system shows clear evidence of ongoing accretion. The $\mathrm{H} \alpha$ equivalent width in the combined spectrum is $6-8 \AA$. (This is below the $10 \AA$ cutoff typically taken as dividing classical from weak-line T Tauri stars, but since AK Sco's components are $\mathrm{F}$ stars, its $\mathrm{H} \alpha$ line luminosity is greater than that of many cooler classical $\mathrm{T}$ Tauri stars.) If the accretion were taking place only from material in the small circumstellar disks and they were not replenished, they would be quickly depleted. Thus, the accretion diagnostics and infrared spectral energy distribution suggest that the region between circumstellar and circumbinary disks is not completely cleared of material. In fact, only a very small surface density of dust is required in the gap to make it optically thick at near-infrared wavelengths 
(JM97), so perhaps continued accretion from the circumbinary disk populates the inner hole sufficiently to maintain the near-IR excess.

However, the system notably does not show the quasi-periodic variability seen in DQ Tau, where enhanced accretion in phase with the binary orbit is thought to arise from material that is periodically pulled from the circumbinary disk at apastron and streams across the binary orbit to land on the stars when they are near periastron (Mathieu et al. 1997). The orbital eccentricity, period, and disk mass of AK Sco are all similar to those in DQ Tau, as are the evidence for continued accretion and the near-power-law spectral energy distribution. Thus, both systems appear not to have completely clear regions between circumstellar and circumbinary disks, and yet their optical variability patterns are quite different. Clearly we have much yet to understand about accretion in the binary environment.

\section{Discussion and Conclusions}

The fact that AK Sco and several other young spectroscopic binaries (DQ Tau and UZ Tau E) do not show evidence of cleared inner holes in their disks, while other systems (such as V4046 Sgr) seem to have completely cleared inner disks, is puzzling. I cannot resolve this puzzle at present, but in closing I note two things about the current data and the models put forth to explain them.

First, it appears that some flow from circumbinary to circumstellar disks occurs in all young binaries with disks. Even V4046 Sgr, which has a remarkably clean inner hole (Figure 2), shows evidence of strong accretion (JM97).

Second, it may be that the diversity of observed disk clearing simply reflects the amount of material that is able to flow from the circumbinary disk onto the stars' circumstellar disks. The amount of flow across a gap may depend on several factors, including binary orbital eccentricity and mass ratio (Artymowicz $\&$ Lubow 1996). Since we have such a small sample of known pre-main-sequence short-period binaries, it is hard to draw general conclusions about exactly which factors are the most important. Thus, the resolution of this puzzle (like many others!) awaits further observational data.

Acknowledgments. I gratefully acknowledge the support of the National Science Foundation's Life in Extreme Environments program through grant AST 97-14246, and I thank Luiz Paulo Vaz for sharing his photometry of AK Sco.

\section{References}

Andersen, J., Lindgren, H., Hazen, M. L., \& Mayor, M. 1989, A\&A, 219, 142

Artymowicz, P., \& Lubow, S. H. 1994, ApJ, 421, 651

Artymowicz, P., \& Lubow, S. H. 1996, ApJ, 467, L77

Favata, F., Micela, G., Sciortino, S., D'Antona, F. 1998, A\&A, 335, 218

Jensen, E. L. N., \& Mathieu, R. D. 1997, AJ, 114, 301

Mathieu, R. D., Stassun, K., Basri, G., Jensen, E. L. N., Johns-Krull, C. M., Valenti, J. A., \& Hartmann, L. W. 1997, AJ, 113, 1841 

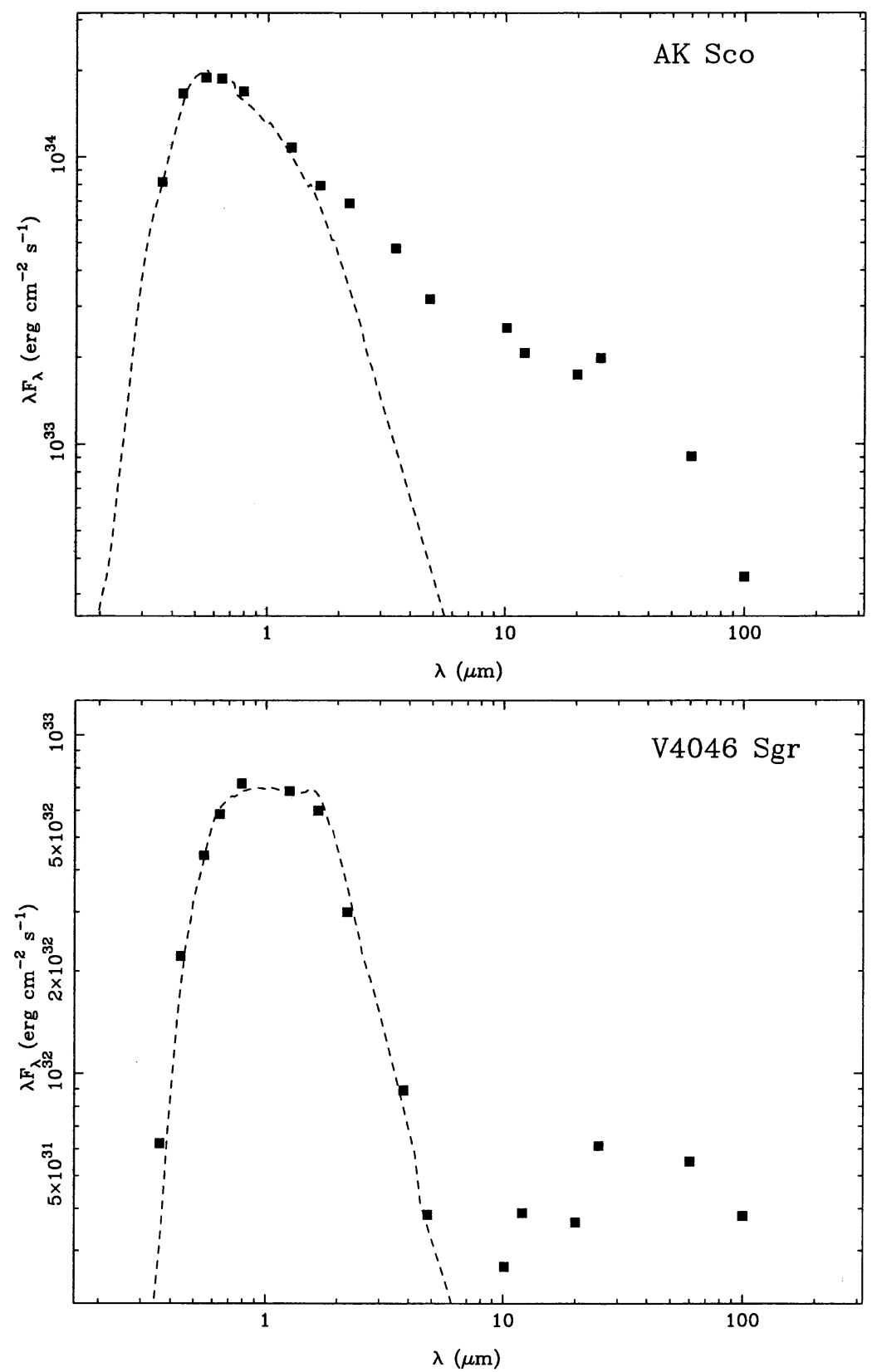

Figure 2. Spectral energy distributions (SEDs) of young spectroscopic binaries; the dashed line shows a model photosphere. Top: The SED of AK Sco is almost a power law, giving no indication of cleared regions in the disk. Bottom: This contrasts markedly with the SED of V4046 Sgr, which shows only photospheric emission out to $\lambda=5 \mu \mathrm{m}$ but an IR excess at longer wavelengths, consistent with a hole in its disk cleared by the binary. (Adapted from Jensen \& Mathieu 1997.) 